\title{
Nonvolatile, reversible electric-field controlled switching of remanent magnetization in multifunctional ferromagnetic/ferroelectric hybrids
}

\author{
A. Brandlmaier, ${ }^{1, \text { a) }}$ S. Geprägs, ${ }^{1}$ G. Woltersdorf, ${ }^{2}$ R. Gross, ${ }^{1,3}$ and S. T. B. Goennenwein ${ }^{1,3}$ \\ ${ }^{1}$ Walther-Meißner-Institut, Bayerische Akademie der Wissenschaften, 85748 Garching, Germany \\ ${ }^{2}$ Institut für Experimentelle und Angewandte Physik, Universität Regensburg, 93040 Regensburg, Germany \\ ${ }^{3}$ Physik-Department E23, Technische Universität München, 85748 Garching, Germany
}

(Received 1 May 2011; accepted 7 July 2011; published online 23 August 2011)

\begin{abstract}
In spin-mechanics, the magnetoelastic coupling in ferromagnetic/ferroelectric hybrid devices is exploited in order to realize an electric-voltage control of magnetization orientation. To this end, different voltage-induced elastic strain states are used to generate different magnetization orientations. In our approach, we take advantage of the hysteretic expansion and contraction of a commercial piezoelectric actuator as a function of electrical voltage to deterministically select one of two electro-remanent elastic strain states. We investigate the resulting magnetic response in a nickel thin film/piezoelectric actuator hybrid device at room temperature, using simultaneous magnetooptical Kerr effect and magnetotransport measurements. The magnetic properties of the hybrid can be consistently described in a macrospin model, i.e., in terms of a single magnetic domain. At zero external magnetic field, the magnetization orientation in the two electro-remanent strain states differs by $15^{\circ}$, which corresponds to a magnetoresistance change of $0.5 \%$. These results demonstrate that the spin-mechanics scheme indeed enables a nonvolatile electrically read- and writable memory bit where the information is encoded in a magnetic property. (C) 2011 American Institute of Physics. [doi:10.1063/1.3624663]
\end{abstract}

\section{INTRODUCTION}

The control of magnetic degrees of freedom via electric fields is a long-standing experimental challenge.$^{1-3}$ A promising approach to this end are composite-type multifunctional structures. Particularly attractive are artificially assembled ferromagnetic/ferroelectric hybrid systems, since they enable an elastic strain-mediated electric-field control of magnetism via inverse magnetostriction. ${ }^{4-9}$ In such hybrids, the control of the key magnetic properties, such as coercive field, saturation magnetization, or remanent magnetization via electric voltages has been demonstrated recently. ${ }^{10-16} \mathrm{~A}$ further major development is the demonstration of a reversible, all-electricfield control of magnetization orientation or reversal, and thus of an electrically controlled magnetization switching. ${ }^{17-26}$ However, the literature so far is focused on a nonhysteretic all-electrical magnetization manipulation, i.e., the electricfield induced magnetization changes are volatile in the sense that they return to their initial state upon removing the electric field. Obviously, the next step now is to achieve a nonvolatile or remanent electric-field control of $\mathbf{M}$. First experiments in this direction have already been performed in Ni thin film/ferroelectric hybrid samples. ${ }^{26,27}$ Here we show an all-electricfield nonvolatile control of remanent magnetization in zero applied magnetic field in ferromagnetic thin film/piezoelectric actuator hybrid systems. ${ }^{20,28-30}$ The basic idea behind the experiments is to exploit the intrinsically hysteretic strainvoltage curve of the piezoelectric actuator to deterministically generate two different elastic strain states at zero applied voltage. Due to the intimate mechanical connection between actu-

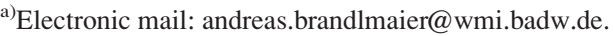

ator and ferromagnet, these two remanent elastic strain states are transferred via magnetoelastic coupling into two distinct remanent magnetization states. We determine the magnetization state both optically and electrically both as a function of magnetic field and electrical voltage applied to the piezoelectric actuator. We find that all our data can be consistently modeled in a macrospin type of approach evidencing two distinct nonvolatile remanent magnetization orientations enclosing an angle of $15^{\circ}$-deterministically and reversibly adjustable via the application of appropriate voltage pulses at zero external magnetic field. Contrary to, e.g., ferroelectric random access memory, ${ }^{31}$ the mechanism utilized here does not rely on a macroscopic polarization reversal of the ferroelectric compound, so that our concept is operational well below the ferroelectric coercive fields.

\section{EXPERIMENT}

The ferromagnetic thin film/piezoelectric actuator hybrid structures investigated here consist of commercially available $\mathrm{Pb}\left(\mathrm{Zr}_{x} \mathrm{Ti}_{1-x}\right) \mathrm{O}_{3}(\mathrm{PZT})$ piezoelectric stack actuators "PSt 150/ $2 \times 3 / 5$ " (Piezomechanik München), onto which $100 \mathrm{~nm}$ thick ferromagnetic nickel (Ni) thin films were deposited using electron beam evaporation at a base pressure of $4.0 \times 10^{-8}$ mbar, followed by $5 \mathrm{~nm}$ Au in situ to prevent oxidation. To enable an electrical readout of the magnetization orientation, electrical contacts in van der Pauw geometry are wire bonded to the Ni film [Fig. 1(a)]. We chose Ni as a prototype ferromagnet with a high Curie temperature $T_{\mathrm{C}}=627 \mathrm{~K}$ (Ref. 32) and bulk saturation magnetization $M_{\mathrm{s}}=493 \mathrm{kA} / \mathrm{m}$ (Ref. 33), a considerable polycrystalline volume magnetostriction $\bar{\lambda}=\frac{2}{5} \lambda_{100}+\frac{3}{5} \lambda_{111}=-32.9 \times 10^{-6}$ (Ref. 34) and anisotropic magnetoresistance (AMR) ratio $\Delta \rho / \rho_{0}=2 \%$ (Ref. 35). 

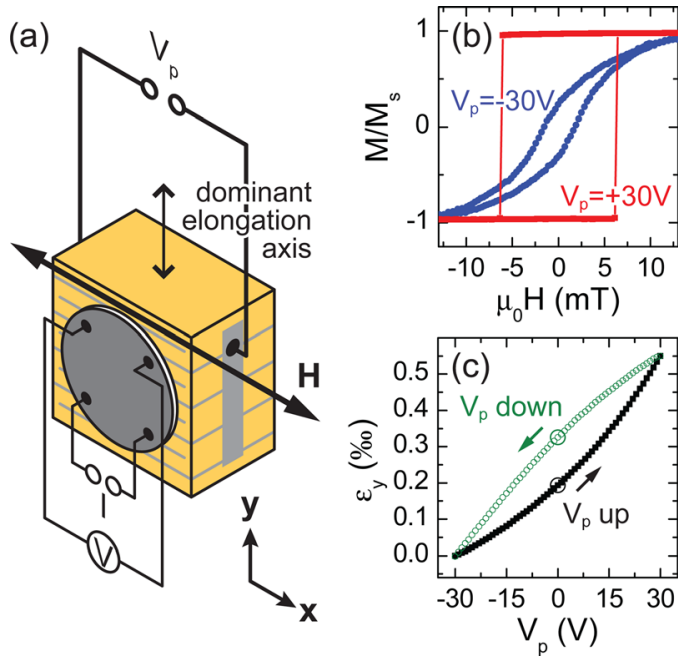

FIG. 1. (Color online) (a) Schematic illustration of the ferromagnetic thin film/actuator hybrid with the contact scheme in van der Pauw geometry. The external magnetic field $\mathbf{H}$ is oriented along $\mathbf{x}$ for all measurements. (b) $M(H)$ loops recorded at fixed voltages $V_{\mathrm{p}}$ showing a magnetically easy loop for $V_{\mathrm{p}}=+30 \mathrm{~V}$ (red squares) and a hard loop for $V_{\mathrm{p}}=-30 \mathrm{~V}$ (blue circles) along $\mathbf{x}$. (c) Hysteretic strain-voltage curve of the actuator showing that the strain $\epsilon_{y}\left(V_{\mathrm{p}}\right)$ exhibits two distinct strain states at $V_{\mathrm{p}}=0 \mathrm{~V}$ (big open circles) depending on $V_{\mathrm{p}}$ history.

Moreover, the magnetoelastic coupling scheme is highly efficient in the polycrystalline Ni films, since the Ni film plane is a magnetically isotropic easy plane, so that voltage-controlled, strain-induced magnetic anisotropies have a substantial impact on the magnetization orientation. ${ }^{29}$

Upon the application of a positive (negative) voltage $V_{\mathrm{p}}$, the actuator expands (contracts) along the dominant elongation direction $\mathbf{y}$ [Fig. 1(a)] with a nominal maximum strain $\epsilon_{y}=1.3 \times 10^{-3}$ in the full voltage swing $-30 \mathrm{~V} \leq V_{\mathrm{p}}$ $\leq+150 \mathrm{~V}$. Due to the elastic properties of the actuator, this deformation results in a compressive or tensile strain in the Ni film along the in-plane direction $\mathbf{x}$ [Fig. 1(a)] for $V_{\mathrm{p}}>0 \mathrm{~V}$ and $V_{\mathrm{p}}<0 \mathrm{~V}$, respectively. Considering the magnetostriction constant $\bar{\lambda}<0$ and the elastic stiffness constants $c_{11}>c_{12}$ of $\mathrm{Ni}^{34}$ the magnetoelastic coupling results in a magnetic easy axis along $\mathbf{x}$ for $V_{\mathrm{p}}>0 \mathrm{~V}$, and accordingly an easy axis along $\mathbf{y}$ and thus a hard axis along $\mathbf{x}$ for $V_{\mathrm{p}}<0 \mathrm{~V}$ (Ref. 20).

To determine the static magnetic properties of the $\mathrm{Ni}$ thin film/actuator hybrid we use longitudinal magneto-optical Kerr effect (MOKE) measurements, which detect the projection $M=\mathbf{M} \cdot \mathbf{x}$ of the magnetization onto the magnetic field direction along $\mathbf{H}=H \mathbf{x}$. The incident light from a cwdiode laser hereby is focused on the center of the Ni film onto a spot with a diameter of about $100 \mu \mathrm{m}$. We simultaneously recorded the magnetoresistance in four-point measurements, with a constant bias current $\mathbf{I}$ flowing along $\mathbf{x}$. All data shown in the following were taken at room temperature.

Figure 1(b) demonstrates the concept of voltage-controlled magnetization-orientation manipulation via elastic strain transfer. The normalized MOKE loops correspond to $\mathbf{M} \cdot \mathbf{x} / M_{\mathrm{s}}$, with the saturation magnetization $M_{\mathrm{s}}$, and were recorded as a function of the external magnetic field magnitude at constant voltages $V_{\mathrm{p}}=+30 \mathrm{~V}$ and $V_{\mathrm{p}}=-30 \mathrm{~V}$, respectively. For $V_{\mathrm{p}}=+30 \mathrm{~V}$, we obtain a rectangular- shaped $M(H)$ loop (full red squares) characteristic of a magnetically easy axis along $\mathbf{x}$, while the elongated, s-shaped $M(H)$ loop (full blue circles) for $V_{\mathrm{p}}=-30 \mathrm{~V}$ indicates a hard direction along $\mathbf{x}$. Figure 1(c) depicts the strain $\epsilon_{y}\left(V_{\mathrm{p}}\right)$ exerted along $\mathbf{y}$ measured using a strain gauge in the voltage range of $-30 \mathrm{~V} \leq V_{\mathrm{p}} \leq+30 \mathrm{~V}$ for a $V_{\mathrm{p}}$ upsweep (full black symbols) and downsweep (open green symbols). The actuator expansion and contraction is hysteretic, as typically observed in ferroelectric PZT. As the maximum applied voltage is below the ferroelectric coercive field of $E_{\mathrm{c}} \approx 4.5 \mathrm{kV} / \mathrm{cm}$ corresponding to $V_{\mathrm{p}} \approx 45 \mathrm{~V}$, the macroscopic polarization of the ferroelectric compound is not switched and only domain-wall motion is induced. The corresponding irreversible displacement contributions generate the observed hysteresis. ${ }^{36}$ In particular, the hysteretic $\epsilon_{y}\left(V_{\mathrm{p}}\right)$ curve allows us to select one of two distinctly different, remanent elastic strain states at zero applied electric field [big open circles in Fig. 1(c)] depending on the voltage history. Via magnetoelastic coupling this is transferred into two distinct remanent magnetization-orientation states at $V_{\mathrm{p}}=0 \mathrm{~V}$ in the adjacent ferromagnet.

\section{RESULTS AND DISCUSSION}

Figures 2(a)-2(d) show how the magnetic properties change with the hysteretic expansion and contraction of the

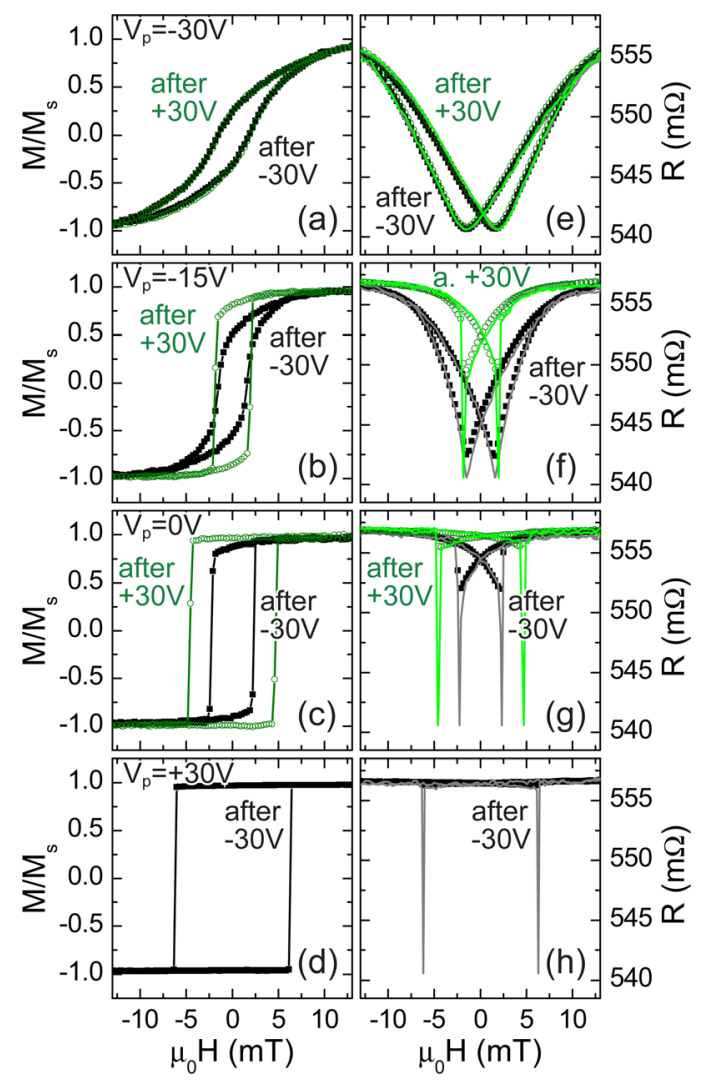

FIG. 2. (Color online) (a)-(d) $M(H)$ loops at fixed $V_{\mathrm{p}}$ for a $V_{\mathrm{p}}$ upsweep from $V_{\mathrm{p}}=-30 \mathrm{~V}$ to $V_{\mathrm{p}}=+30 \mathrm{~V}$ (full black squares) and a subsequent $V_{\mathrm{p}}$ downsweep (open green circles). The lines are guides to the eye. (e)-(h) Simultaneously recorded $R(H)$ loops (symbols). The solid lines display simulations of the AMR in a single-domain model showing very good overall agreement with the experiment. The mechanical hysteresis of the actuator results in significant differences between $V_{\mathrm{p}}$ up- and downsweep in the MOKE loops $[(b),(c)]$, which is also reflected in the AMR curves $[(f),(g)]$. 
actuator as a function of $V_{\mathrm{p}}$. The full black squares depict the MOKE $M(H)$ loops for a $V_{\mathrm{p}}$ upsweep, recorded at fixed voltages $V_{\mathrm{p}}=-30 \mathrm{~V},-15 \mathrm{~V}, 0 \mathrm{~V}$, and $+30 \mathrm{~V}$, as shown in Figs. 2(a), 2(b), 2(c), and 2(d), respectively, whereas open green circles depict the corresponding $M(H)$ loops for a $V_{\mathrm{p}}$ downsweep. The lines are guides to the eye. According to the discussion above [cf. Fig. 1(b)], the $M(H)$ loop is magnetically hard at $V_{\mathrm{p}}=-30 \mathrm{~V}$. The loop becomes increasingly easy with increasing $V_{\mathrm{p}}$, until it is perfectly rectangularshaped at $V_{\mathrm{p}}=+30 \mathrm{~V}$. Upon gradually decreasing $V_{\mathrm{p}}$ again, the loop becomes gradually harder and finally perfectly coincides with the corresponding $V_{\mathrm{p}}$ upsweep loop [Fig. 2(a)]. At the intermediate voltages [Figs. 2(b) and 2(c)] we observe a considerable difference between the loops recorded for the same $V_{\mathrm{p}}$ value in a $V_{\mathrm{p}}$ up- and downsweep, owing to the hysteretic strain-voltage curve of the actuator. The resistance curves displayed in Figs. 2(e)-2(h), recorded simultaneously to the $M(H)$ loops, show that the AMR of the Ni film also characteristically changes depending on the $V_{\mathrm{p}}$ history, which evidently yields characteristic differences between the $R(H)$ loops for the $V_{\mathrm{p}}$ up- and downsweeps [Figs. 2(f) and $2(\mathrm{~g})]$. This demonstrates that the magnetization state can be directly readout electrically.

We next show that the voltage-induced strain affects only the magnetization orientation, but not its magnitude. To this end, we extract the macrospin magnetization orientation $\cos \alpha=M / M_{\mathrm{s}}$ with respect to the current direction along $\mathbf{x}$ from the $M(H)$ loops. Obviously, this is valid only in a macrospin approximation (no magnetic domain formation). We then calculate the longitudinal AMR using the macrospin expression $R=R_{\perp}+\left(R_{\|}-R_{\perp}\right) \cos ^{2} \alpha$ (Ref. 35), where $R_{\perp}$ and $R_{\|}$are the resistance values for $\mathbf{I} \perp \mathbf{M}$ and $\mathbf{I} \| \mathbf{M}$, respectively. Using $R_{\|}=557.5 \mathrm{~m} \Omega$ and $R_{\perp}=540.6 \mathrm{~m} \Omega$, we obtain the solid lines in Figs. 2(e)-2(h). Except for a narrow region (less than $1 \mathrm{mT}$ ) around the magnetic coercive fields $H_{\mathrm{c}}$, where sharp dips appearing in the simulation are not observed in the corresponding experiment, the simulations are in very good agreement with the experimental data. In order to model the magnetization-reversal process, coherent rotation and domain-wall nucleation and/or unpinning usually are combined. ${ }^{37}$ Coherent rotation relies on a continuous rotation of a single homogeneous magnetic domain. The sharp, irreversible features around the magnetic coercive fields often are attributed to domain-wall effects. It furthermore has been shown (e.g., in Ref. 38) that the magnetization reversal in a ferromagnetic thin film with uniaxial anisotropy is caused by coherent rotation when the external magnetic field is oriented close to the magnetic hard axis [cf. Figs. 2(a) and 2(e)], while for magnetic-field orientations close to an easy axis [and thus for increasingly abrupt changes of the magnetization orientation in the $M(H)$ loops in Fig. 2] domain-wall processes take over in the vicinity of the magnetic switching fields. Hence, for increasing $V_{\mathrm{p}}$ one expects that our simple single-domain macrospin modeling will fail to adequately describe the experiments close to $H_{\mathrm{c}}$-which accounts for the deviations between experiment and simulation at the switching fields in Figs. 2(f), 2(g), and 2(h). For all other magnetic-field values, however, the overall good agreement demonstrates that we can describe the voltage controlled magnetization modifications in good approximation as a coherent magnetization reorientation in a single-domain (macrospin) model. The coincidence furthermore shows that $M(H)$ and $R(H)$ measurements yield equivalent information about the magnetization orientation. Note that the information on the magnetization state obtained from MOKE and the AMR measurements is in quite remarkable agreement, considering the fact that the $\mathrm{Ni}$ area laterally probed by these two techniques $\left(\approx 0.008 \mathrm{~mm}^{2}\right.$ for MOKE and $\approx 3 \mathrm{~mm}^{2}$ for AMR) differs by more than two orders of magnitude.

To quantitatively evaluate the characteristic differences in the $M(H)$ loop as a function of $V_{\mathrm{p}}$ evident from Figs. 2(a)2(d), we have plotted both the coercive field $\mu_{0} H_{\mathrm{c}}$ and the normalized remanent magnetization $M_{\mathrm{r}} / M_{\mathrm{S}}$ observed experimentally in Figs. 3(a) and 3(b), respectively. For both quantities the values for increasing and decreasing $V_{\mathrm{p}}$ are clearly different. In particular, two distinctly different states in $M_{\mathrm{r}}$, i.e., at $\mu_{0} H=0 \mathrm{mT}$ and $V_{\mathrm{p}}=0 \mathrm{~V}$ are observed, as indicated by the big open circles. This opens the perspective of a nonvolatile, all-voltage controlled remanent magnetization control. To demonstrate that the magnetization orientation can indeed be remanently altered by solely varying $V_{\mathrm{p}}$ while keeping $\mu_{0} H$ constant, we also recorded $M$ and $R$ as a function of $V_{\mathrm{p}}$ at zero external magnetic field. Prior to the $V_{\mathrm{p}}$ sweep, we prepared the magnetization into a well-defined initial state along the easy axis by setting the voltage to $V_{\mathrm{p}}=+30 \mathrm{~V}$ and applying a saturating magnetic field
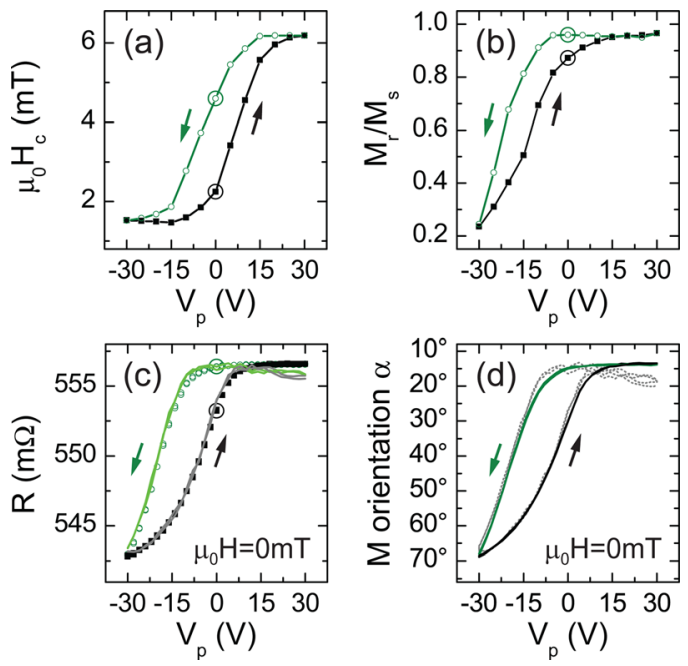

FIG. 3. (Color online) Coercive field $\mu_{0} H_{\mathrm{c}}$ (a) and normalized remanent magnetization $M_{\mathrm{r}} / M_{\mathrm{s}}$ (b) extracted from the $M(H)$ loops shown in Figs. 2(a)-2(d). Both quantities exhibit the hysteretic behavior characteristic of the mechanoelastic hysteresis of the actuator. (c) Two consecutive $R\left(V_{\mathrm{p}}\right)$ loops recorded at $\mu_{0} H=0 \mathrm{mT}$, after preparing a single-domain magnetic state. A single voltage cycle starts at $V_{\mathrm{p}}=+30 \mathrm{~V}$, followed by a $V_{\mathrm{p}}$ downsweep (open green circles) and a subsequent upsweep (full black squares). The curves also consistently show a hysteretic behavior. The solid lines depict the AMR calculated based on the corresponding $M\left(V_{\mathrm{p}}\right)$ loops, showing very good agreement with the experimental AMR data. (d) Macrospin magnetization orientation $\alpha$, calculated from the measured AMR data displayed in (c) with the AMR parameters given in the text (solid black and green lines), and from the measured MOKE data (dashed gray lines). At $V_{\mathrm{p}}=0 \mathrm{~V}, \mathbf{M}$ can be reversibly switched between two magnetization orientation states, which corresponds to a $\mathbf{M}$ reorientation of $\Delta \alpha=15^{\circ}$. In the full voltage range $-30 \mathrm{~V} \leq V_{\mathrm{p}} \leq+30 \mathrm{~V}, \mathbf{M}$ can be reversibly adjusted within $55^{\circ}$. 
$\mu_{0} H=+120 \mathrm{mT}$. We then swept the magnetic field to $\mu_{0} H=0 \mathrm{mT}$ and kept it constant at this value, and consecutively recorded two complete $R\left(V_{\mathrm{p}}\right)$ voltage cycles $V_{\mathrm{p}}=+30 \mathrm{~V} \rightarrow-30 \mathrm{~V} \rightarrow+30 \mathrm{~V}$. The $R\left(V_{\mathrm{p}}\right)$ loops thus obtained are shown in Fig. 3(c) (open green circles and full black squares again indicate the $V_{\mathrm{p}}$ down- and upsweep, respectively). This corroborates the results displayed in Fig. $3(\mathrm{~b})$, as a $V_{\mathrm{p}}$ sweep at fixed $\mu_{0} H=0 \mathrm{mT}$ also yields a hysteretic behavior with two distinctly different magnetic states at $V_{\mathrm{p}}=0 \mathrm{~V}$. We observe a considerable total AMR change from $V_{\mathrm{p}}=+30 \mathrm{~V}$ to $-30 \mathrm{~V}$ of $[R(+30 \mathrm{~V})-R(-30 \mathrm{~V})] /$ $R(-30 \mathrm{~V})=2.5 \%$, and a change between the two states at $V_{\mathrm{p}}=0 \mathrm{~V}$ of $[R(0 \mathrm{~V}$, down $)-R(0 \mathrm{~V}$, up $)] / R(0 \mathrm{~V}$, up $)=0.6 \%$. Moreover, as the two $R\left(V_{\mathrm{p}}\right)$ cycles perfectly coincide, the voltage controlled magnetization rotation appears to be fully reversible. Using the $M\left(V_{\mathrm{p}}\right)$ loops recorded simultaneously with $R\left(V_{\mathrm{p}}\right)$, we again simulate the AMR with the parameters given above. The AMR curves thus calculated are displayed by solid lines in Fig. 3(c). Evidently, the measured and simulated AMR data are in very good agreement, which again corroborates the validity of the macrospin approach and shows that the $\mathbf{M}$ orientation derived from resistance measurements is fully equivalent to that obtained from direct magnetization measurements. This consistency furthermore proves the robustness of the single-domain model, as in the MOKE setup the light beam only probes a $\sim 100 \mu \mathrm{m}$ diameter region of the Ni film, whereas the four-point resistance measurement integrally probes the magnetic film. A quantitative analysis of the $M\left(V_{\mathrm{p}}\right)$ and $R\left(V_{\mathrm{p}}\right)$ data is shown in Fig. $3(\mathrm{~d})$, which depicts the macrospin magnetization orientation $\alpha$, calculated from the measured AMR data displayed in Fig. 3 (c) with the values of the parameters $R_{\|}$and $R_{\perp}$ given above (solid black and green lines), and from the measured MOKE data (dashed gray lines). At $V_{\mathrm{p}}=0 \mathrm{~V}$, we thus obtain a $\mathbf{M}$ orientation $\alpha\left(V_{\mathrm{p}, \text { down }}=0 \mathrm{~V}\right)=15^{\circ}$ for the $V_{\mathrm{p}}$ downsweep and $\alpha\left(V_{\mathrm{p} \text {,up }}=0 \mathrm{~V}\right)=30^{\circ}$ for the $V_{\mathrm{p}}$ upsweep. In the total voltage swing $-30 \mathrm{~V} \leq V_{\mathrm{p}} \leq+30 \mathrm{~V}$, we can reversibly rotate the magnetization orientation by $55^{\circ}\left[\alpha\left(V_{\mathrm{p}}=+30 \mathrm{~V}\right)=14^{\circ}\right.$ and $\alpha\left(V_{\mathrm{p}}=-30 \mathrm{~V}\right)=69^{\circ}$. Taken together, our observations show that the magnetization orientation at $V_{\mathrm{p}}=0 \mathrm{~V}$ and $\mu_{0} H=0 \mathrm{mT}$ can be reversibly switched between two distinct states enclosing an angle of $15^{\circ}$ only via $V_{\mathrm{p}}$ sweeps, while keeping $\mu_{0} H=0 \mathrm{mT}$ fixed. This enables a voltage controlled, nonvolatile magnetization "switching", as will be discussed in the following.

The remanent voltage control of $\mathbf{M}$ is illustrated in Fig. 4 . We start with a preparation of the magnetization in a single domain state along a magnetic easy axis at $V_{\mathrm{p}}=+30 \mathrm{~V}$. To normalize the MOKE magnetization data recorded subsequently, we start with the magnetic field at $\mu_{0} H=-120 \mathrm{mT}$ in a negative saturation state [Fig. 4(a)]. After sweeping the magnetic field beyond positive saturation to $\mu_{0} H=+120 \mathrm{mT}$, we finally set the field back to $\mu_{0} H=0 \mathrm{mT}$ and keep it constant at this value (point A). The subsequent data acquisition at $\mu_{0} H=0 \mathrm{mT}$ is illustrated in Fig. 4(b). We apply the voltage sequences $V_{\mathrm{p}}=0 \mathrm{~V} \rightarrow+30 \mathrm{~V} \rightarrow 0 \mathrm{~V}$ (red lines in the upper half of the panel) and $V_{\mathrm{p}}=0 \mathrm{~V} \rightarrow-30 \mathrm{~V} \rightarrow 0 \mathrm{~V}$ (blue lines) to switch the magnetization into one of the two distinct magnetic states determined by the remanent elastic strain, and
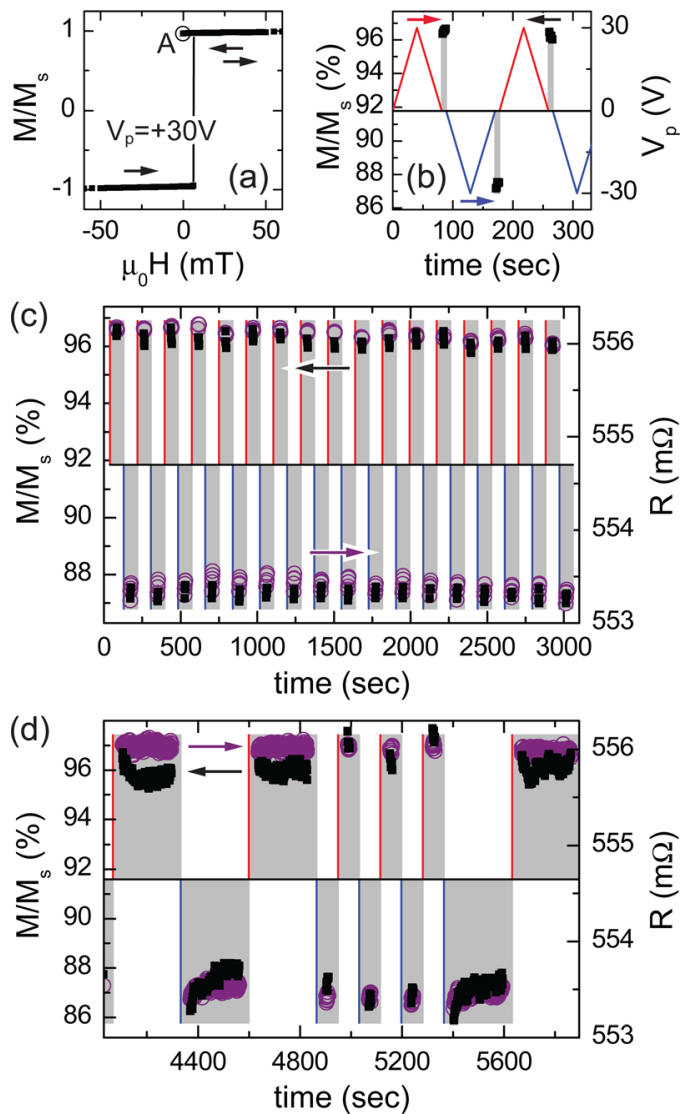

FIG. 4. (Color online) (a) Magnetic preparation sweep at $V_{\mathrm{p}}=+30 \mathrm{~V}$ to establish a well-defined magnetization state. After sweeping from $\mu_{0} H$ $=+120 \mathrm{mT}$ to $0 \mathrm{mT}$ (point A) the magnetic field is kept fixed at $\mu_{0} H=0 \mathrm{mT}$. (b) Illustration of the data acquisition process. The data is recorded at $V_{\mathrm{p}}=0$ $\mathrm{V}$ (gray bar) after applying a voltage sequence either $V_{\mathrm{p}}=0 \mathrm{~V} \rightarrow+30 \mathrm{~V} \rightarrow 0$ $\mathrm{V}$ (red line) or $V_{\mathrm{p}}=0 \mathrm{~V} \rightarrow-30 \mathrm{~V} \rightarrow 0 \mathrm{~V}$ (blue line). (c) Demonstration of repeated electro-elasto-magnetic switching processes, with $M$ (full black symbols) and $R$ (open purple symbols) being recorded five times in each acquisition window (gray). (d) Electro-elasto-magnetic memory-bit response for other acquisition windows.

then simultaneously record $M$ [full black symbols in Fig. 4(b)] and $R$ at $V_{\mathrm{p}}=0 \mathrm{~V}$. Both $M\left(V_{\mathrm{p}}=0 \mathrm{~V}\right)$ and $R\left(V_{\mathrm{p}}=0 \mathrm{~V}\right)$ are readout five times per acquisition cycle, which is indicated with a gray bar. We would like to emphasize again that after the initial magnetic preparation sweep, the magnetic field is kept constant at $\mu_{0} H=0 \mathrm{mT}$ during the whole data-recording process. The $M\left(V_{\mathrm{p}}=0 \mathrm{~V}\right)$ (full black symbols) and $R\left(V_{\mathrm{p}}=0 \mathrm{~V}\right)$ (open purple symbols) data depicted in Fig. 4(c) for a number of switching processes within a total time of about 50 min clearly show that the magnetization can be reversibly and deterministically switched between two distinct orientations and therefore conclusively demonstrate a nonvolatile electric field-control of remanent magnetization with applied voltages below the ferroelectric coercive field. Figure 4(d) presents a part of the measurement sequence with a higher number of readings per acquisition cycle displaying in total each $1600 M\left(V_{\mathrm{p}}=0 \mathrm{~V}\right)$ and $R\left(V_{\mathrm{p}}=0 \mathrm{~V}\right)$ readings within an acquisition time of $30 \mathrm{~min}$. These two distinct magnetic states at $V_{\mathrm{p}}=0 \mathrm{~V}$ are clearly retained. Note that, however, the MOKE signal tends to drift as a function of time probably owing to small temperature fluctuations, while the resistance signal in all our 
measurements proved very stable. Overall, these findings conceptionally demonstrate the feasibility of a spin-mechanics memory with all-electrical read and write at zero external magnetic field, which allows for room-temperature operation, and at electric field strengths well below the ferroelectric coercive fields.

\section{CONCLUSION}

In summary, we have demonstrated an all-electric field, remanent control of magnetization orientation in ferromagnetic thin film/piezoelectric actuator hybrid structures at zero external magnetic field. We find that appropriate voltage cycles with magnitudes well below the ferroelectric coercive fields applied to the piezoelectric actuator allow to deterministically switch the magnetization orientation in the ferromagnetic film at $V_{\mathrm{p}}=0 \mathrm{~V}$ and $\mu_{0} H=0 \mathrm{mT}$ between two remanent orientations differing by an angle of $15^{\circ}$. We exploit the hysteretic strain-voltage curves of the actuator in subcoercive voltage loops, which yields two distinctly different strain states at $V_{\mathrm{p}}=0 \mathrm{~V}$. The strain is transferred into the ferromagnet and results in a magnetization modification via magnetoelastic coupling (spin-mechanics scheme). From simultaneously recorded $R(H)$ and $M(H)$ loops, we find that $R$ can be accurately modeled in a macrospin (single-domain) type of approach. This shows that domain formation is not an issue (i) at least on the length scale of the MOKE spot and/or because (ii) the straininduced changes in the free-energy surface of the Ni thin film are too small to account for the domain-wall formation energy. ${ }^{38}$ Hence, the voltage-controlled magnetization modifications are due to a rotation of the magnetization orientation. Changing solely $V_{\mathrm{p}}$ at fixed magnetic field corroborates this notion, as we observe a reversible $M\left(V_{\mathrm{p}}\right)$ and $R\left(V_{\mathrm{p}}\right)$ control also exhibiting two distinct magnetization orientation states at $V_{\mathrm{p}}=0 \mathrm{~V}$ enclosing an angle of $15^{\circ}$, depending on the $V_{\mathrm{p}}$ history. This enables an all-voltage controlled, nonvolatile magnetization control at $\mu_{0} H=0 \mathrm{mT}$, by which the magnetization can be reversibly and deterministically switched between two distinct orientations. Our findings provide clear evidence for the versatility of the spin-mechanical magnetization-control scheme and open interesting perspectives, e.g., for applications in spintronic data-storage devices, where multifunctional hybrid systems can be employed as strain-based nonvolatile solid-state memory bits.

\section{ACKNOWLEDGMENTS}

Financial support via DFG Project No. GO 944/3-1 and the German Excellence Initiative via the "Nanosystems Initiative Munich (NIM)" are gratefully acknowledged.

\footnotetext{
${ }^{1}$ M. Fiebig, J. Phys. D: Appl. Phys. 38, R123 (2005).

${ }^{2}$ W. Eerenstein, N. D. Mathur, and J. F. Scott, Nature 442, 759 (2006).

${ }^{3}$ R. Ramesh and N. A. Spaldin, Nat. Mater. 6, 21 (2007).
}

${ }^{4}$ W. Eerenstein, M. Wiora, J. L. Prieto, J. F. Scott, and N. D. Mathur, Nat. Mater. 6, 348 (2007).

${ }^{5}$ C.-W. Nan, M. I. Bichurin, S. Dong, D. Viehland, and G. Srinivasan, J. Appl. Phys. 103, 031101 (2008).

${ }^{6}$ C. Thiele, K. Dörr, O. Bilani, J. Rödel, and L. Schultz, Phys. Rev. B 75, 054408 (2007).

${ }^{7}$ C. Israel, N. D. Mathur, and J. F. Scott, Nat. Mater. 7, 93 (2008).

${ }^{8}$ S. Sahoo, S. Polisetty, C.-G. Duan, S. S. Jaswal, E. Y. Tsymbal, and C. Binek, Phys. Rev. B 76, 092108 (2007).

${ }^{9}$ F. Zavaliche, H. Zheng, L. Mohaddes-Ardabili, S. Y. Yang, Q. Zhan, P. Shafer, E. Reilly, R. Chopdekar, Y. Jia, P. Wright, D. G. Schlom, Y. Suzuki, and R. Ramesh, Nano Lett. 5, 1793 (2005).

${ }^{10}$ J.-W. Lee, S.-C. Shin, and S.-K. Kim, Appl. Phys. Lett. 82, 2458 (2003).

${ }^{11}$ H. Boukari, C. Cavaco, W. Eyckmans, L. Lagae, and G. Borghs, J. Appl. Phys. 101, 054903 (2007).

${ }^{12}$ N. Moutis, D. Suarez-Sandoval, and D. Niarchos, J. Magn. Magn. Mater. 320, 1050 (2008).

${ }^{13}$ C. Israel, S. Kar-Narayan, and N. D. Mathur, Appl. Phys. Lett. 93, 173501 (2008).

${ }^{14}$ Y. Chen, J. Gao, T. Fitchorov, Z. Cai, K. S. Ziemer, C. Vittoria, and V. G. Harris, Appl. Phys. Lett. 94, 082504 (2009).

${ }^{15}$ J. Wang, J. Hu, H. Wang, H. Jiang, Z. Wu, J. Ma, X. Wang, Y. Lin, and C. W. Nan, J. Appl. Phys. 107, 083901 (2010).

${ }^{16}$ Z. Li, J. Wang, Y. Lin, and C. W. Nan, Appl. Phys. Lett. 96, 162505 (2010).

${ }^{17}$ S.-K. Kim, J.-W. Lee, S.-C. Shin, H. W. Song, C. H. Lee, and K. No, J. Magn. Magn. Mater. 267, 127 (2003).

${ }^{18}$ C. Cavaco, M. van Kampen, L. Lagae, and G. Borghs, J. Mater. Res. 22, 2111 (2007).

${ }^{19}$ Y. Chen, A. L. Geiler, T. Fitchorov, C. Vittoria, and V. G. Harris, Appl. Phys. Lett. 95, 182501 (2009).

${ }^{20}$ M. Weiler, A. Brandlmaier, S. Geprägs, M. Althammer, M. Opel, C. Bihler, H. Huebl, M. S. Brandt, R. Gross, and S. T. B. Goennenwein, New J. Phys. 11, 013021 (2009).

${ }^{21}$ J. J. Yang, Y. G. Zhao, H. F. Tian, L. B. Luo, H. Y. Zhang, Y. J. He, and H. S. Luo, Appl. Phys. Lett. 94, 212504 (2009).

${ }^{22}$ Y. Chen, T. Fitchorov, C. Vittoria, and V. G. Harris, Appl. Phys. Lett. 97, 052502 (2010).

${ }^{23}$ Y. Chen, T. Fitchorov, A. Geiler, J. Gao, C. Vittoria, and V. Harris, Appl. Phys. A 100, 1149 (2010).

${ }^{24}$ J. Ma, Y. Lin, and C. W. Nan, J. Phys. D: Appl. Phys. 43, 012001 (2010).

${ }^{25}$ Y. Zhang, J. Liu, X. H. Xiao, T. C. Peng, C. Z. Jiang, Y. H. Lin, and C. W. Nan, J. Phys. D: Appl. Phys. 43, 082002 (2010).

${ }^{26}$ S. Geprägs, A. Brandlmaier, M. Opel, R. Gross, and S. T. B. Goennenwein, Appl. Phys. Lett. 96, 142509 (2010).

${ }^{27}$ T. Wu, A. Bur, P. Zhao, K. P. Mohanchandra, K. Wong, K. L. Wang, C. S. Lynch, and G. P. Carman, Appl. Phys. Lett. 98, 012504 (2011).

${ }^{28}$ A. Brandlmaier, S. Geprägs, M. Weiler, A. Boger, M. Opel, H. Huebl, C. Bihler, M. S. Brandt, B. Botters, D. Grundler, R. Gross, and S. T. B. Goennenwein, Phys. Rev. B 77, 104445 (2008).

${ }^{29}$ C. Bihler, M. Althammer, A. Brandlmaier, S. Geprägs, M. Weiler, M. Opel, W. Schoch, W. Limmer, R. Gross, M. S. Brandt, and S. T. B. Goennenwein, Phys. Rev. B 78, 045203 (2008).

${ }^{30}$ S. T. B. Goennenwein, M. Althammer, C. Bihler, A. Brandlmaier, S. Geprägs, M. Opel, W. Schoch, W. Limmer, R. Gross, and M. S. Brandt, Phys. Status Solidi (RRL) 2, 96 (2008).

${ }^{31}$ A. Kingon, Nature 401, 658 (1999).

${ }^{32}$ J. M. Leger, C. Loriers-Susse, and B. Vodar, Phys. Rev. B 6, 4250 (1972).

${ }^{33}$ R. Pauthenet, J. Appl. Phys. 53, 2029 (1982).

${ }^{34}$ E. W. Lee, Rep. Prog. Phys. 18, 184 (1955).

${ }^{35}$ T. McGuire and R. Potter, IEEE Trans. Magn. 11, 1018 (1975).

${ }^{36}$ D. Bolten, U. Böttger, and R. Waser, J. Appl. Phys. 93, 1735 (2003).

${ }^{37}$ J. M. Florczak and E. D. Dahlberg, Phys. Rev. B 44, 9338 (1991).

${ }^{38}$ S.-s. Yan, W. J. Liu, J. L. Weston, G. Zangari, and J. A. Barnard, Phys. Rev. B 63, 174415 (2001). 